University of New Orleans

ScholarWorks@UNO

2-1983

\title{
Single-reflection film-substrate half-wave retarders with nearly stationary reflection properties over a wide range of incidence angles
}

\author{
R. M.A. Azzam \\ University of New Orleans, razzam@uno.edu \\ M. Emdadur Rahman Khan
}

Follow this and additional works at: https://scholarworks.uno.edu/ee_facpubs

Part of the Electrical and Electronics Commons, and the Physics Commons

\section{Recommended Citation}

R. M. A. Azzam and M. Emdadur Rahman Khan, "Single-reflection film-substrate half-wave retarders with nearly stationary reflection properties over a wide range of incidence angles," J. Opt. Soc. Am. 73, 160-166 (1983)

This Article is brought to you for free and open access by the Department of Electrical Engineering at ScholarWorks@UNO. It has been accepted for inclusion in Electrical Engineering Faculty Publications by an authorized administrator of ScholarWorks@UNO. For more information, please contact scholarworks@uno.edu. 


\title{
Single-reflection film-substrate half-wave retarders with nearly stationary reflection properties over a wide range of incidence angles
}

\author{
R. M. A. Azzam and M. Emdadur Rahman Khan*
}

Department of Electrical Engineering, University of New Orleans, Lakefront, New Orleans, Louisiana 70148

Received August 26, 1982

\begin{abstract}
The complex reflection coefficient for the $p$ polarization of a transparent film on an absorbing or transparent substrate can be made equal to the negative of that for the $s$ polarization, and hence the film-substrate system acts as a half-wave retarder (HWR), by proper selection of film refractive index $N_{1}$, film thickness $d$, and angle of incidence $\phi$. This condition, which generally holds only at normal incidence, becomes possible at oblique incidence also if $N_{1}$ is within a certain range, $1<N_{1}<\hat{N}_{1}$. For a given substrate and given $N_{1}$, a procedure is described to determine $\phi_{\mathrm{HWR}}$ and $d_{\mathrm{HWR}}$ that achieve a HWR. As $N_{1}$ is increased from 1 to the upper limit $\hat{N}_{1}, \phi_{\mathrm{HWR}}$ decreases from $90^{\circ}$ to 0 monotonically. $d_{\mathrm{HWR}}$ approximately equals (exactly equals when the substrate is a perfect dielectric or a perfect conductor) an odd multiple of half of the film-thickness period evaluated at $\phi_{\mathrm{HWR}}$. Significantly, we find that the film-substrate HWR retains nearly the same characteristics of normal-incidence reflection over the range of angle from 0 up to (and beyond) $\phi_{\mathrm{HWR}}$. Detailed data are presented of HWR's that use transparent films on metallic (Al and $\mathrm{Ag})$, semiconducting $(\mathrm{Si})$, and dielectric (glass) substrates at two laser wavelengths $(0.6328$ and $10.6 \mu \mathrm{m})$. Film-substrate HWR's permit the realization of simple polarization-insensitive parallel-mirror beam displacers, $90^{\circ}$ rooftop reflectors, and biconical axicons and waxicons.
\end{abstract}

\section{INTRODUCTION}

Retarders are devices that introduce a differential phase shift between orthogonally linearly polarized ${ }^{1}$ components of light with no or insignificant accompanying differential amplitude attenuation. Classically, their operation is based on natural or induced linear birefringence in certain media or on the difference between the $p$ - and $s$-reflection phase shifts associated with total internal reflection. ${ }^{2-4}$ Recently, external reflection from an optically isotropic film-covered substrate was demonstrated to be a suitable basis for the design of new retarders. $^{5-9}$ The natural extension of this idea to multilayer-coated reflection retarders has also been accomplished. ${ }^{10-12}$ The latter devices have very high (near-unity) reflectance at the expense of added complexity and cost.

We remain interested primarily in single-reflection filmsubstrate retarders because of their simplicity. Previous work ${ }^{5-8}$ in this area seems to indicate that it is not possible to achieve half-wave $\left(180^{\circ}\right)$ retardation by a single obliqueincidence reflection from a film-substrate system. Such $180^{\circ}$ retardation is obtained at normal incidence (by this or any other optically isotropic surface) and, as such, is not particularly useful. ${ }^{13}$

In this paper we show that the foregoing limitation can be lifted by the proper choice of refractive index $N_{1}$ of the transparent film. In particular, we find that, for a given substrate (be it metallic or otherwise), if $N_{1}$ is selected in a certain range, $1<N_{1}<\hat{N}_{1}$, the film-substrate system will act as an exact half-wave retarder (HWR) for a certain (reduced) film thickness $d_{\text {HWR }}$ at a certain angle of oblique incidence $\phi_{\mathrm{HWR}}$. As $N_{1}$ is increased from 1 to its upper limit $\hat{N}_{1}, \phi_{\mathrm{HWR}}$ decreases monotonically from $90^{\circ}$ to 0 . For $N_{1}>\hat{N}_{1}, \phi_{\mathrm{HWR}}$ stays equal to 0 , which is the condition that we have encountered before. ${ }^{5-8}$ Significantly, we also find that, for all angles of incidence between 0 and $\phi_{\mathrm{HWR}}$ (and a little beyond $\phi_{\mathrm{HWR}}$ ), the film-substrate system has nearly stationary reflection characteristics; hence it performs as a wide-angle HWR.

Section 2 describes the design procedure of film-substrate HWR's. In Section 3, the procedure is applied to HWR's that use dielectric films on an $\mathrm{Al}$ substrate for $\mathrm{He}-\mathrm{Ne}$ laser light of wavelength $\lambda=0.6328 \mu \mathrm{m}$. Results for HWR's based on dielectric (glass) and semiconducting ( $\mathrm{Si}$ ) substrates (at $\lambda=$ $0.6328 \mu \mathrm{m}$ ), which are at least academically interesting, and others that use a nearly perfect conductor $\left(\mathrm{Ag}\right.$ at the $\mathrm{CO}_{2}$-laser wavelength $\lambda=10.6 \mu \mathrm{m}$ ) are presented in Section 4 . Finally, in Section 5 we point out important applications of the filmsubstrate HWR in polarization-insensitive beam displacers, axicons, rooftop reflectors, and waxicons.

\section{DESIGN PROCEDURE OF HALF-WAVE RETARDERS}

The complex amplitude-reflection coefficients of a filmsubstrate system for light that is linearly polarized parallel $(p)$ or perpendicular $(s)$ to the plane of incidence are given by $^{3}$

$$
\begin{aligned}
R_{\nu} & =\frac{r_{01 \nu}+r_{12 \nu} X}{1+r_{01 \nu} r_{12 \nu} X}, \quad \nu=p, s, \\
X & =e^{-j 2 \pi \zeta},
\end{aligned}
$$

$r_{i j \nu}$ is Fresnel's complex reflection coefficient of the $i j$ interface for the $\nu$ polarization and

$$
\zeta=d / D_{\phi}
$$


is the normalized film thickness, where

$$
D_{\phi}=\frac{\lambda}{2}\left(N_{1}^{2}-N_{0}^{2} \sin ^{2} \phi\right)^{-1 / 2}
$$

is the angle-of-incidence-dependent film-thickness period of the periodic reflection coefficients as functions of $d . \lambda$ is the free-space wavelength of light, and $N_{0}$ and $N_{1}$ are the real refractive indices of the medium of incidence ( 0 ) and the film (1), respectively, that are assumed to be transparent. The substrate (2) is, in general, absorbing, and its refractive index $\mathrm{N}_{2}$ is complex. In the following we will assume that the medium of incidence is air (or vacuum), as is commonly the case, and set $N_{0}=1$. The design procedure, however, is the same for any transparent medium of incidence.

For a given film-substrate system to operate as a HWR at oblique incidence, the condition

$$
R_{p}=-R_{s}
$$

must be satisfied. Substitution of Eqs. (1) into Eq. (5) leads to a quadratic equation in $X$,

$$
P X^{2}+Q X+U=0,
$$

with coefficients given by

$$
P=\sigma_{01} \beta_{12}, \quad Q=\left(1+\beta_{01}\right) \sigma_{12}, \quad U=\sigma_{01},
$$

where $\beta_{i j}$ and $\sigma_{i j}$ represent the product and the sum, respectively, of the $p$ and $s$ Fresnel reflection coefficients of the $i j$ interface:

$$
\beta_{i j}=r_{i j p} r_{i j s}, \quad \sigma_{i j}=r_{i j p}+r_{i j s} .
$$

Equation (6) is complex and can be broken down, by taking its real and imaginary parts, into two real equations in two real unknowns: the angle of incidence $\phi$ and the reduced normalized film thickness $\zeta, 0 \leq \zeta<1$. If $(\phi, \zeta)$ is a solution of Eq. (6), then $(\phi, \zeta+m)$, where $m$ is an integer, is also a solution because $X$ is a periodic function of $\zeta$ with period 1 .

The two nonlinear equations that are equivalent to Eq. (6) can be solved for $(\phi, \zeta)$ by using, for example, the NewtonRaphson method. This, however, requires simultaneous iteration on both $\phi$ and $\zeta$ in search of the solution. We have followed another simpler and more-elegant procedure that separates the determination of $\phi$ and $\zeta$. In this method, which is similar to one used in Refs. 5 and 6, only $\phi$ is determined by iteration, and $\zeta$ is subsequently obtained explicitly. This can be achieved by solving Eq. (6) for $X$,

$$
X=\left[-Q \pm\left(Q^{2}-4 P U\right)^{1 / 2}\right] / 2 P,
$$

and requiring that

$$
|X|=1 \text {, }
$$

which follows from Eq. (2) and from the assumptions that the film is transparent and that $N_{1}>N_{0} \sin \phi$, which is satisfied when $N_{0}=1$. Equation (10), where $X$ is determined by the right-hand side of Eq. (9), can be solved for $\phi$ as its only unknown. Recall that $P, Q$, and $U$ are determined by the interface Fresnel reflection coefficients, Eqs. (7) and (8), which, in turn, are functions of the angle of incidence $\phi$. Once $\phi$ has been determined, the right-hand side of Eq. (9), i.e., complex $X$, can be evaluated. Subsequently, the normalized film thickness $\zeta$ is obtained from Eq. (2) as

$$
\zeta=(-1 / 2 \pi) \arg X \text {. }
$$

All possible film thicknesses for a HWR are given by

$$
d_{m}=(\zeta+m) D_{\phi},
$$

where $m$ is an integer. In the following we will consider only the least (reduced) normalized and actual film thicknesses, $0 \leq \zeta<1$ and $0 \leq d<D_{\phi}$. The solution of Eq. (6) that leads to a HWR is identified by $\left(\phi_{\mathrm{HWR}}, \zeta_{\mathrm{HWR}}\right)$.

An important characteristic of a HWR is its intensity reflectance $\mathscr{R}_{\mathrm{HWR}}$, which is the same for the $p$ and the $s$ (hence for all) polarizations. It is calculated from Eqs. (1), at $\phi=$ $\phi_{\mathrm{HWR}}$ and $\zeta=\zeta_{\mathrm{HWR}}$, as

$$
\mathcal{R}_{\mathrm{HWR}}=\left|R_{p}\right|^{2}=\left|R_{s}\right|^{2} .
$$

We will examine the performance of a given HWR as a function of angle of incidence $\left(\phi \neq \phi_{\mathrm{HWR}}\right)$ as described by the ratio of complex reflection coefficients,

$$
\rho=R_{p} / R_{s}=|\rho| e^{j \Delta},
$$

where $|\rho|$ reprsents the relative amplitude attenuation and $\Delta$ is the relative phase shift, which are equal to 1 and $180^{\circ}$, respectively, when $\phi=\phi_{\mathrm{HWR}}$ and $\zeta=\zeta_{\mathrm{HWR}}$. In addition, the deviation of $\rho$ of a HWR from -1 , caused by small shifts of the film thickness trom the design value $d_{\mathrm{HWR}}$, is determined.

\section{DIELECTRIC FILM-ALUMINUM SUBSTRATE HALF-WAVE RETARDERS AT $\lambda=$ $0.6328 \mu \mathrm{m}$}

The design procedure of Section 2 was applied to a system that consists of a transparent film of adjustable refractive index $N_{1}$ on an Al substrate of complex refractive index ${ }^{14} N_{2}=1.212$ $-j 6.924$ at the $\mathrm{He}-\mathrm{Ne}$-laser wavelength $\lambda=0.6328 \mu \mathrm{m}$. For an assumed value of $N_{1}>1$, a solution is sought for Eq. (10) and hence for Eq. (6). Figure 1 shows $|X|-1$ versus $\phi$ for $N_{1}$ $=1.38$, which corresponds to a $\mathrm{MgF}_{2}$ film. ${ }^{15}$ Two curves appear that correspond to the + and - (the two values of the complex square root) in Eq. (9). Only one curve intersects the $\phi$ axis once at $\phi_{\mathrm{HWR}}=34.43^{\circ}$. From Eq. (11), we determine the least normalized film thickness, $\zeta_{\mathrm{HWR}}=0.49622$. At $\phi_{\mathrm{HWR}}$ Eq. (4) gives the film-thickness period $D \phi_{\mathrm{HWR}}=$ $0.25133 \mu \mathrm{m}$, and from Eq. (12) (with $m=0$ ) we obtain the least film thickness $d_{\mathrm{HWR}}=0.12472 \mu \mathrm{m}$. The reflectance of the HWR, calculated from Eq. (13), is $\mathscr{R}_{\mathrm{HWR}}=0.8393$. As a check on the solution, the ratio of complex reflection coefficients $\rho$ [Eq. (4)] is evaluated at ( $\left.\phi_{\mathrm{HWR}}, \zeta_{\mathrm{HWR}}\right)$ and is found to differ from -1 by less than $10^{-7}$ in real and imaginary parts.

Figure 2 shows (for the $\mathrm{MgF}_{2}-\mathrm{Al}$ system at $\lambda=0.6328 \mu \mathrm{m}$ ) three closed contours traced by $\rho(\phi, \zeta)$ in the complex plane obtained when $\phi$ is fixed at $\phi_{\mathrm{HWR}}$ and $\phi_{\mathrm{HWR}} \pm 5^{\circ}$ and $\zeta$ is varied over one period from 0 to 1 . The contour for $\phi_{\mathrm{HWR}}$ passes through the point $\rho=-1$, as expected, and this occurs when $\zeta=\zeta_{\text {HWR }}$. The nature of these so-called constantangle-of-incidence contours (CAIC's) of the ellipsometric function $\rho$ differs significantly from that described in Ref. 5 . For the $\mathrm{SiO}_{2}-\mathrm{Si}$ system ${ }^{5}$ at $\lambda=0.6328 \mu \mathrm{m}$ (and at several other wavelengths ${ }^{8}$ ), every CAIC for $0<\phi<\phi_{s}$, where $\phi_{s}$ is the $s$ polarizing angle, encloses the point $\rho=-1$. That is why film-substrate HWR's were thought to be possible only at normal incidence. Here we have shown that a CAIC for $\phi>$ 0 may pass through, instead of enclose, the point $\rho=-1$, 


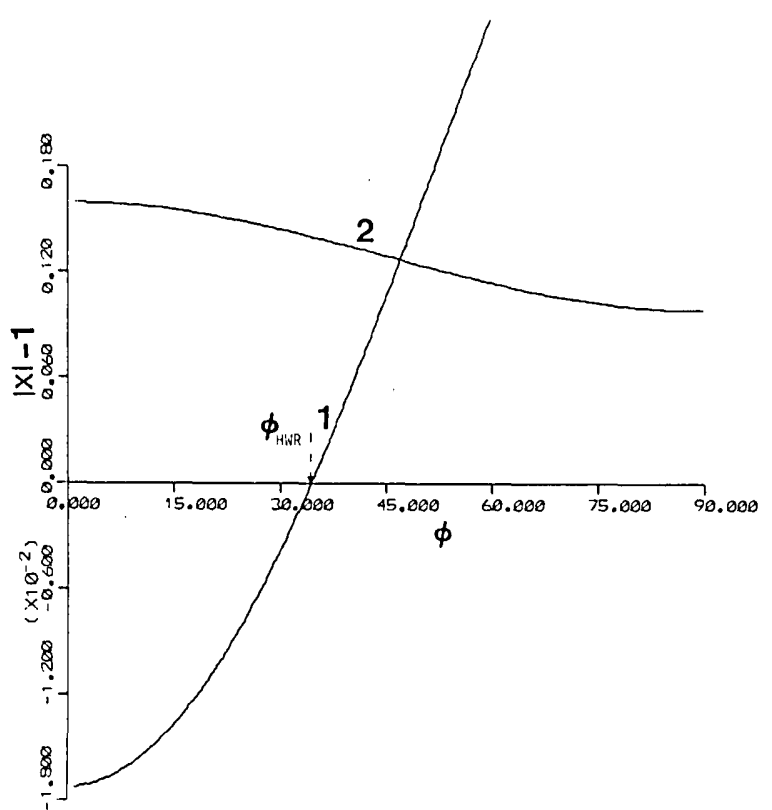

Fig. 1. Finding a solution of Eq. (10) for a $\mathrm{MgF}_{2}-\mathrm{Al}$ film-substrate system at $\lambda=0.6328 \mu \mathrm{m} .|X|-1$ versus $\phi$ has two distinct branches 1 and 2 that correspond to the + and - in Eq. (9). Only branch 1 intersects the $\phi$ axis at an angle $\phi_{\mathrm{HWR}}=34.43^{\circ}$ at which the filmsubstrate system can operate as a HWR. Curves 1 and 2 follow the different lower and upper scales of the ordinate axis, respectively, with the portion of curve 1 above the $\phi$ axis following an extension of the lower scale.

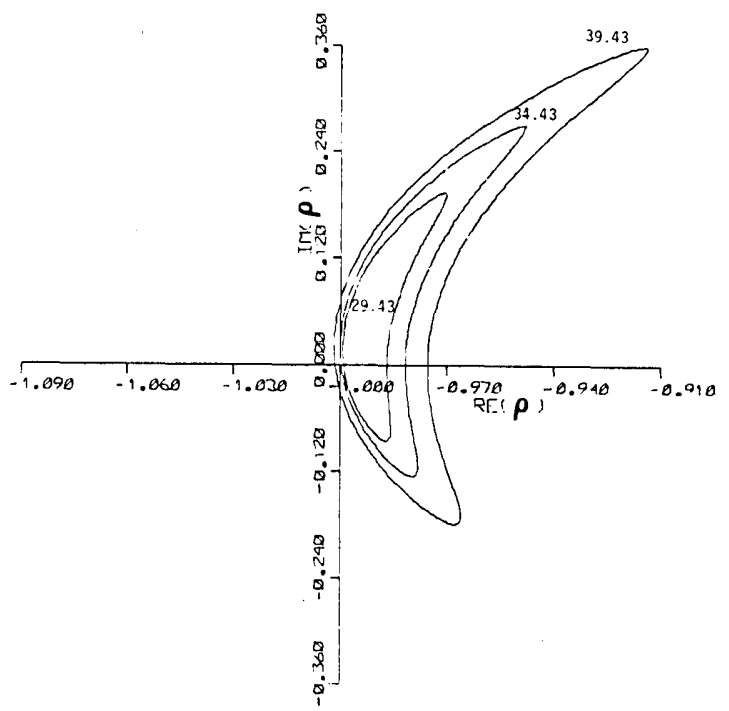

Fig. 2. CAIC of the ratio of complex $p$ and $s$ reflection coefficients $\rho$ of the $\mathrm{MgF}_{2}-\mathrm{Al}$ system at $\lambda=0.6328 \mu \mathrm{m}$ for three angles of incidence: $\phi_{\mathrm{HWR}}=34.43^{\circ}$ and $\phi_{\mathrm{HWR}} \pm 5^{\circ}$. The CAIC for $\phi_{\mathrm{HWR}}$ passes through the point $\rho=-1$, as expected, whereas the CAIC for $\phi_{\text {HWR }}$ $-5^{\circ}=29.43^{\circ}$ does not enclose that point.

which means that HWR's are realizable at oblique incidence. In Fig. 2, the CAIC for $\phi_{\mathrm{HWR}}-5^{\circ}$ does not enclose the point $\rho=-1$.

Figure 3(a) shows the complex-plane constant-thickness contour (CTC) of $\rho$ (for the $\mathrm{MgF}_{2}-\mathrm{Al}$ system at $\lambda=0.6328 \mu \mathrm{m}$ ) at $d=d_{\mathrm{HWR}}=0.12472 \mu \mathrm{m}$. As $\phi$ is increased from 0 to $90^{\circ}$, the CTC begins at $\rho=-1$ and ends at $\rho=+1$. A most important feature of the CTC is not visible in Fig. 3(a). We know that the CTC must make a loop as $\phi$ increases from 0 to $\phi_{\mathrm{HWR}}=34.43^{\circ}$ because $\rho=-1$ at these two values of $\phi$. It is interesting and significant that such a loop is small. This loop, which is of insignificant width, exists entirely in the second quadrant of the complex plane and is shown on a magnified scale in Fig. 3(b). This means that $\rho$ essentially dwells at -1 for values of $\phi$ from 0 to (and beyond) $\phi_{\mathrm{HWR}}$ and that this $\mathrm{MgF}_{2}-\mathrm{Al}$ film-substrate system (with $d=d_{\mathrm{HWR}}=$ $0.12472 \mu \mathrm{m})$ acts as a wide-angle HWR.

Figures 4 (a) and 4(b) show $|\rho|$ versus $\phi$ and $\Delta$ versus $\phi$, respectively, along the CTC of Fig. 3 for $0 \leq \phi \leq 45^{\circ}$. $|\rho|$ differs from 1 by less than 0.008 , and $\Delta$ differs from $180^{\circ}$ by less than $1.67^{\circ}$, over this range of angles. This confirms the good wide-angle performance of this HWR.

To verify if other reflection parameters of this HWR may also be essentially stationary with $\phi$, we have plotted in Figs. $5(\mathrm{a})$ and 5(b) the individual $p$ and $s$ reflectances and phase shifts, respectively, versus $\phi$ over the same range $0 \leq \phi \leq 45^{\circ}$. At $\phi=\phi_{\mathrm{HWR}}=34.43^{\circ}$, we have $\mathcal{R}_{p}=\mathcal{R}_{s}$ and $\Delta_{p}-\Delta_{s}=$ $-180^{\circ}$, as expected. This figure confirms that all reflection characteristics of this $\mathrm{MgF}_{2}-\mathrm{Al}$ HWR remain close to their

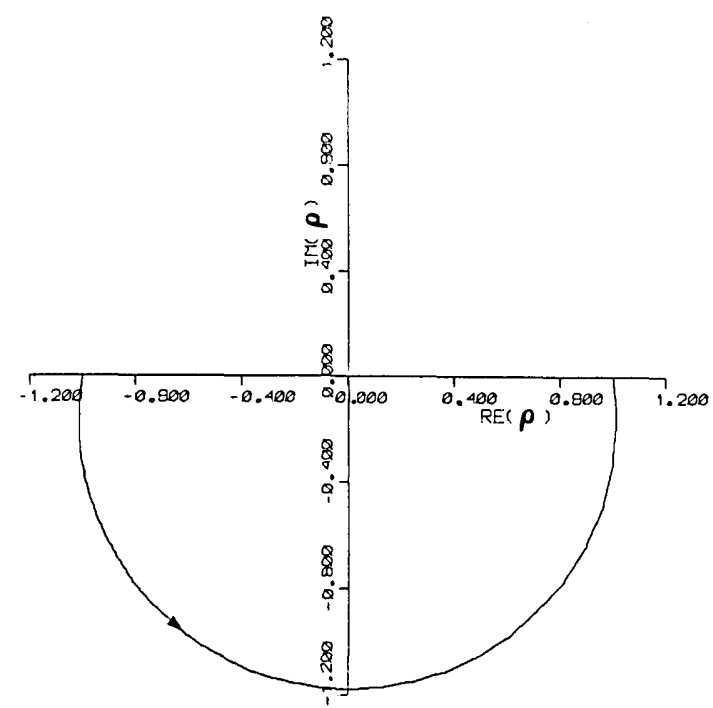

(a)

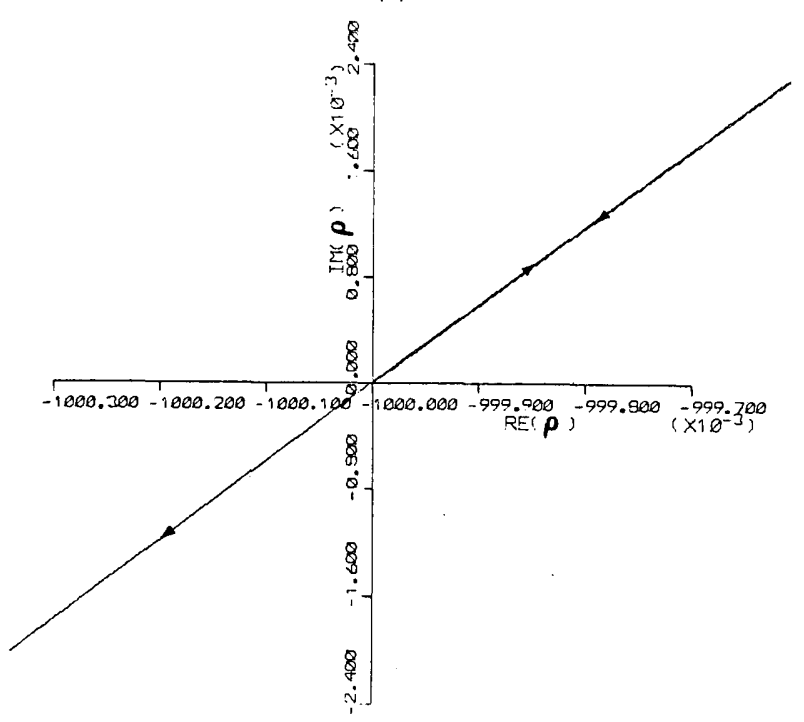

(b)

Fig. 3. (a) CTC of $\rho$ of the $\mathrm{MgF}_{2}-\mathrm{Al}$ system for film thickness $d_{\mathrm{HWR}}$ $=0.12472 \mu \mathrm{m}$. (b) A small loop of the CTC that is invisible in (a). Arrows indicate directions in which the contour is traced as $\phi$ increases from 0 to $90^{\circ}$. 


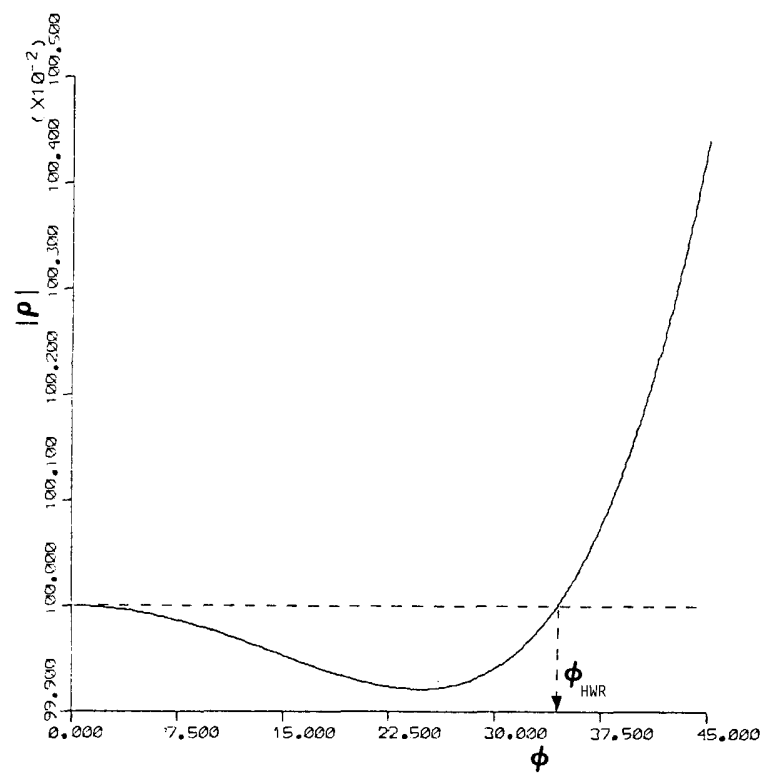

(a)

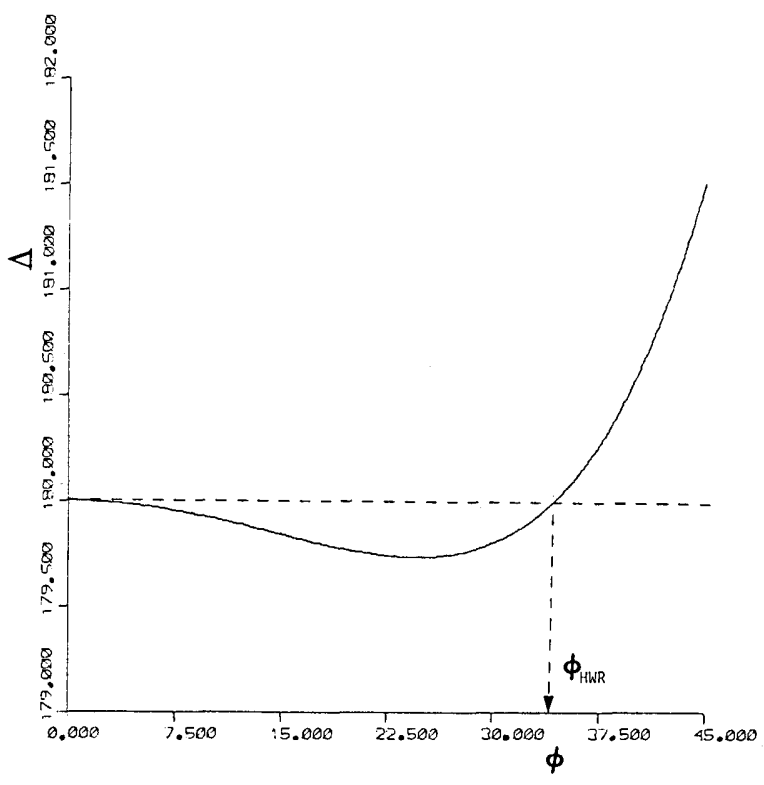

(b)

Fig. 4. (a) $|\rho|$ versus $\phi$ and (b) $\Delta$ versus $\phi, 0 \leq \phi \leq 45^{\circ}$, for a $\mathrm{MgF}_{2}-\mathrm{Al}$ HWR at $\lambda=0.6328 \mu \mathrm{m}$.

normal-incidence values for angles of incidence up to nearly $45^{\circ}$. This important conclusion holds, to a good degree of approximation, for other film-substrate HWR's reported in this paper.

Figure 6 shows the deviation of $|\rho|$ from 1 and the deviation of $\Delta$ from $180^{\circ}$ as the thickness of the $\mathrm{MgF}_{2}$ film is changed by $\pm 100 \AA$ from the correct design value $d_{\mathrm{HWR}}=0.12472 \mu \mathrm{m}$, while keeping $\phi=\phi_{\mathrm{HWR}}=34.43^{\circ}$. This figure indicates acceptable operation as a HWR in the presence of limited thickness control. It can also be interpreted to mean that the $\mathrm{HWR}$ is reasonably insensitive to small wavelength shift around $\lambda=0.6328 \mu \mathrm{m}$ if the effect of material dispersion is ignored.

To explore all possible HWR's that use an Al substrate coated by a transparent film at $\lambda=0.6328 \mu \mathrm{m}$, the design procedure of Section 2 was applied repeatedly for successively increasing discrete values of the film refractive index beginning with $N_{1}$ just slightly above 1 . A solution for a HWR ceases to exist when $N_{1}$ exceeds an upper limit ${ }^{16} N_{1}=\hat{N}_{1} \approx$ 1.46. As $N_{1}$ increases from 1 to $\hat{N}_{1}, \phi_{\text {HWR }}$ decreases monotonically from $90^{\circ}$ to 0 . This is shown in Fig. 7, which also displays all other important characteristics (normalized film thickness $\zeta_{\text {HWR }}$, actual film thickness $d_{\text {HWR }}$, and reflectance $\mathcal{R}_{\mathrm{HWR}}$ versus $\left.\phi_{\mathrm{HWR}}\right)$. Note that $\zeta_{\mathrm{HWR}}, d_{\mathrm{HWR}}$, and $\mathcal{R}_{\mathrm{HWR}}$ all increase monotonically with $\phi_{\text {HWR }}$. It is also significant to note that $\zeta_{\text {HWR }}$ remains close to 0.5 , so that $d_{\text {HWR }}$ equals approximately half of the film-thickness period $D \phi_{\mathrm{HWR}}$.

Although Fig. 7 gives a comprehensive graphical view of all possible design results of HWR's that use a transparent film on an Al substrate at $\lambda=0.6328 \mu \mathrm{m}$, Table 1 gives selected data at five discrete values of film refractive index: $N_{1}=1.05$, $1.15,1.25,1.35,1.45$.

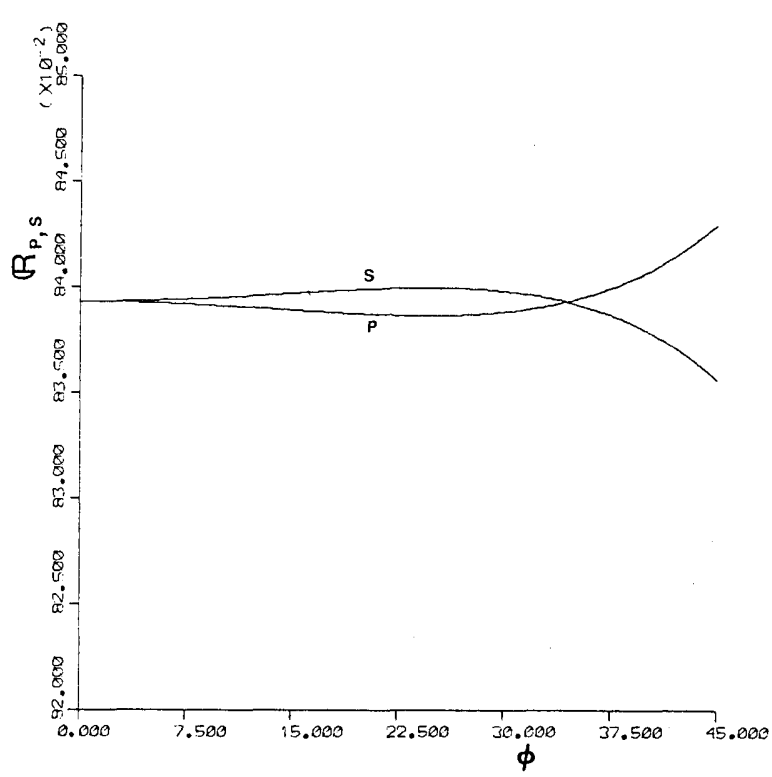

(a)

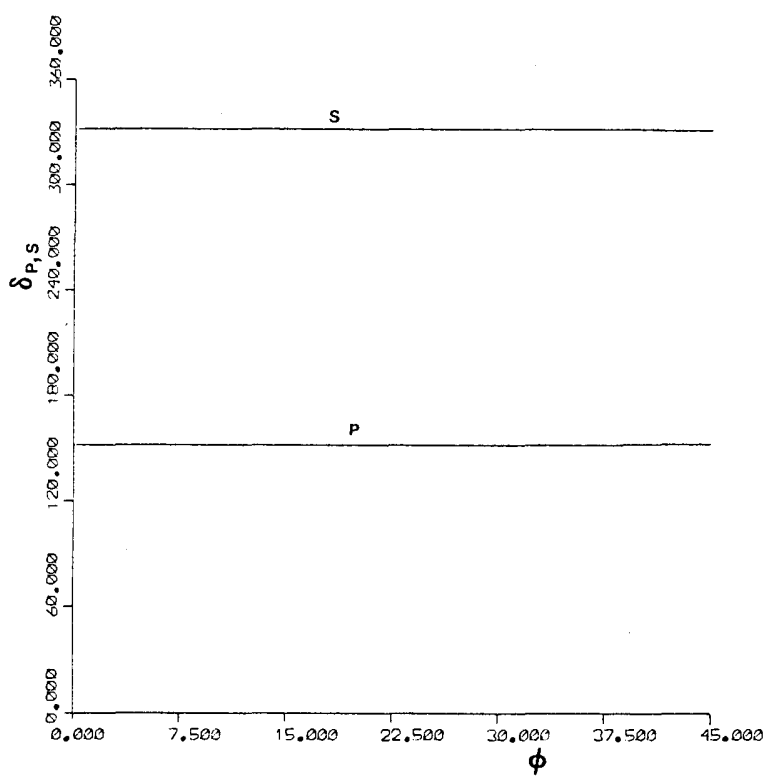

(b)

Fig. 5. (a) $\mathscr{R}_{p}$ and $\mathscr{R}_{s}$ versus $\phi$ and (b) $\delta_{p}$ and $\delta_{s}$ versus $\phi, 0 \leq \phi \leq$ $45^{\circ}$ for a $\mathrm{MgF}_{2}-\mathrm{Al} \mathrm{HWR}$ at $\lambda=0.6328 \mu \mathrm{m}$. 


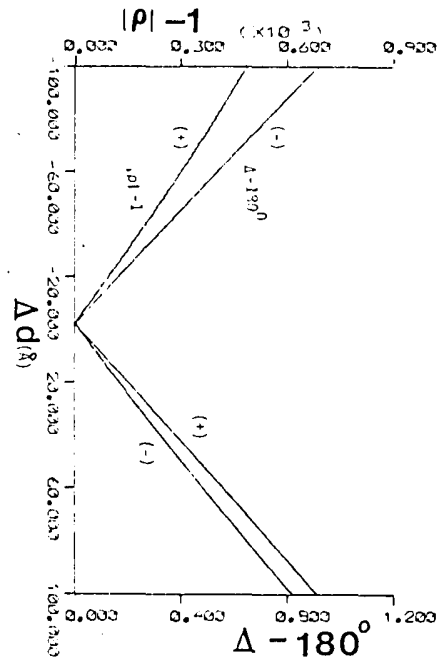

Fig. 6. Magnitude error, $|\rho|-1$, and phase error, $\Delta-180^{\circ}$, caused by changing the thickness of the $\mathrm{MgF}_{2}$ film of a $0.6328-\mu \mathrm{m} \mathrm{MgF} \mathrm{Mgl}_{2}-\mathrm{Al}$ HWR from the correct design value $d_{\mathrm{HWR}}=0.12472 \mu \mathrm{m}$ by $\Delta d$ of up to $\pm 100 \AA$. The + and - marked by the curves give the signs of the errors.

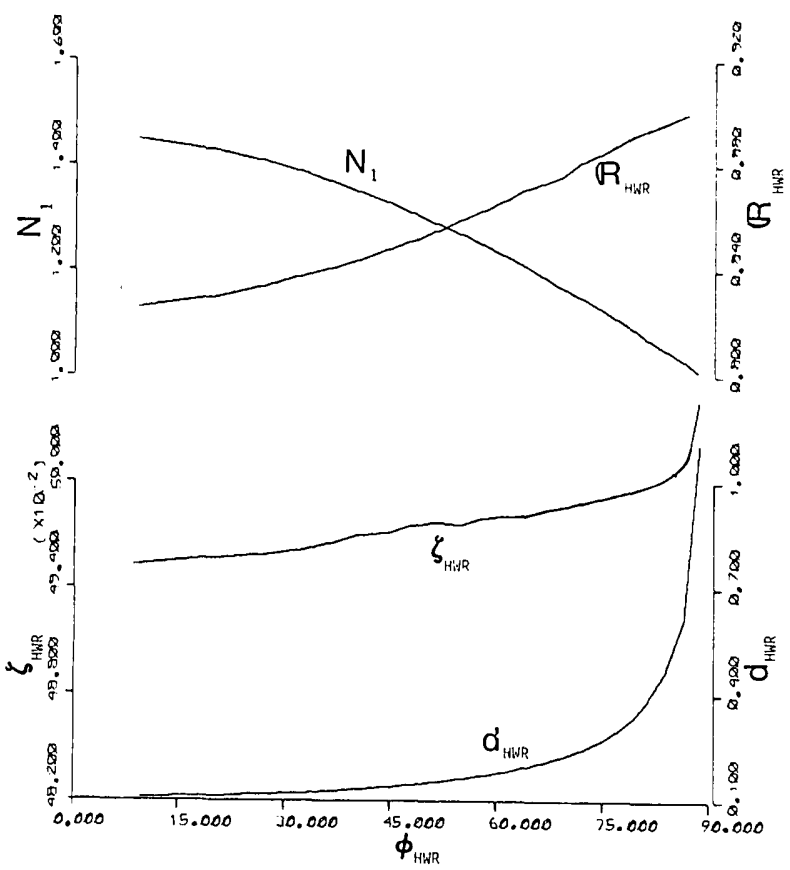

Fig. 7. Comprehensive results for $0.6328-\mu \mathrm{m}$ HWR's that use transparent films on an Al substrate of complex refractive index $\mathrm{N}_{2}$ $=1.212-j 6.924$. Top: film refractive index $N_{1}$ and intensity reflectance $\mathcal{R}_{\text {HWR }}$. Bottom: normalized and actual least film thicknesses $\zeta_{\text {HWR }}$ and $d_{\text {HWR }}$ (in micrometers), respectively. The common abscissa for all curves is the angle of incidence $\phi_{\mathrm{HWR}}$ in degrees.

Figures $8(\mathrm{a})$ and 8 (b) show $|\rho|$ versus $\phi$ and $\Delta$ versus $\phi$, respectively, for the HWR's listed in Table 1 to indicate the performance of these devices over the entire range of incidence angles, $0 \leq \phi \leq 90^{\circ}$. Also shown for reference in Figs. 8(a) and 8(b) are the $|\rho|$ versus $\phi$ and $\Delta$ versus $\phi$ curves for the bare $\mathrm{Al}$ substrate (bottom curves). Prominent effects of the dielectric-layer coatings (which produce HWR's at oblique incidence) are to change the dip of the $|\rho|$ versus $\phi$ curve into a peak and to change the $\Delta$ versus $\phi$ curve from one that decreases from $180^{\circ}$ to 0 monotonically to one that initially decreases from $180^{\circ}$ to a shallow minimum followed by a steep rise to $360^{\circ}$. Also significant to note in Figs. $8(\mathrm{a})$ and $8(\mathrm{~b})$ is how close $|\rho|$ stays near 1 and $\Delta$ stays near $180^{\circ}$ over a wide range of incidence angles for the dielectric-coated $\mathrm{Al}$ HWR's.

Whereas solid thin films of refractive indices less than $\sim 1.25$ (such as those quoted in Table 1) are uncommon in the visible spectrum, the results for $N_{1} \leq 1.25$ are representative of a situation in which the medium of incidence is not air but rather a dense phase $\left(N_{0}>1\right)$.

\section{HALF-WAVE RETARDERS USING OTHER SUBSTRATES}

Figures 9 and 10 present the properties of HWR's that use a transparent film on a glass $\left(N_{2}=1.5\right)$ or $\mathrm{Si}\left(N_{2}=3.85-\right.$ $j 0.02)^{5}$ substrate, respectively, at $\lambda=0.6328 \mu \mathrm{m}$. The results for glass are mainly of academic interest because of the small film refractive index and low reflectance. An interesting point to note in Fig. 9 is that $\zeta=0.5$ exactly for all values of $N_{1}$ (at all $\phi$ ). Thus is true for all transparent substrates, as can be proved analytically. The reflectance for the Si-based HWR's (Fig. 10) is an order of magnitude higher than that achievable with glass, but it is still too small to be useful. In Fig. 10, $\zeta=$ 0.5 almost exactly because of the numerically small $(0.02)$ extinction coefficient of Si.

Figure 11 gives results for single-reflection HWR's that operate at the $\mathrm{CO}_{2}$-laser wavelength $\lambda=10.6 \mu \mathrm{m}$ using a transparent film on a silver substrate $\left(N_{2}=9.5-j 73\right) .^{10}$ The range of film refractive index for HWR operation is $1<N_{1} \lesssim$ 1.42. Materials with low refractive index in the infrared are available. For example, $\mathrm{CaFl}_{2}$ and $\mathrm{LiFl}_{2}$ have refractive indices ${ }^{17}$ of about 1.3 and 1.1 , respectively, at $10.6 \mu \mathrm{m}$. The normalized film thickness $\zeta$ is again very close to 0.5 (it increases from 0.49905 to 0.50016 as $\phi_{\mathrm{HWR}}$ increases from 0 to $90^{\circ}$, respectively). $\zeta=0.5$ holds exactly for a perfectly conducting as well as for a perfectly transparent substrate. Figure 11 shows that all HWR's have the excellent property of an intensity reflectance that is greater than $98.5 \%$. Of course, the origin of this high reflectance of the HWR is the high reflectance of the Ag substrate at $10.6 \mu \mathrm{m}$.

\section{OTHER APPLICATIONS OF THE FILM- SUBSTRATE HALF-WAVE RETARDERS}

Achieving half-wave retardation on single reflection from a film-substrate system provides the basis for the design of parallel-mirror beam displacers and axicons that preserve polarization. In this case the two mirrors of a beam displacer or the two cones of an axicon have identical surfaces made of the same dielectric film of the same thickness on the same

Table 1. Data for Several HWR's That Use Transparent Films on an Al Substrate of Complex Refractive Index $N_{2}=1.212-j 6.924$ at $\lambda=0.6328 \mu \mathrm{m}^{a}$

\begin{tabular}{llllll}
\hline$N_{1}$ & 1.05 & 1.15 & 1.25 & \multicolumn{1}{c}{1.35} & \multicolumn{1}{c}{1.45} \\
\hline$\phi_{\text {HWR }}$ & 84.31 & 72.08 & 58.17 & 40.94 & 8.81 \\
$\zeta_{\text {HWR }}$ & 0.49939 & 0.49861 & 0.49769 & 0.49659 & 0.49529 \\
$D_{\text {HWR }}$ & 0.94403 & 0.48986 & 0.34508 & 0.26806 & 0.21943 \\
$d_{\text {HWR }}$ & 0.47144 & 0.24425 & 0.17174 & 0.13312 & 0.10868 \\
$\Re_{\text {HWR }}$ & 0.8996 & 0.8819 & 0.8636 & 0.8449 & 0.8260 \\
\hline
\end{tabular}

${ }^{a} N_{1}$ is the film refractive index. $\phi, \zeta, D_{\phi}, d$, and $\mathcal{R}$ (with the subscript HWR) represent, respectively, the angle of incidence in degrees, reduced normalized film thickness, film thickness period in micrometers, least film thickness in micrometers, and intensity reflectance. 


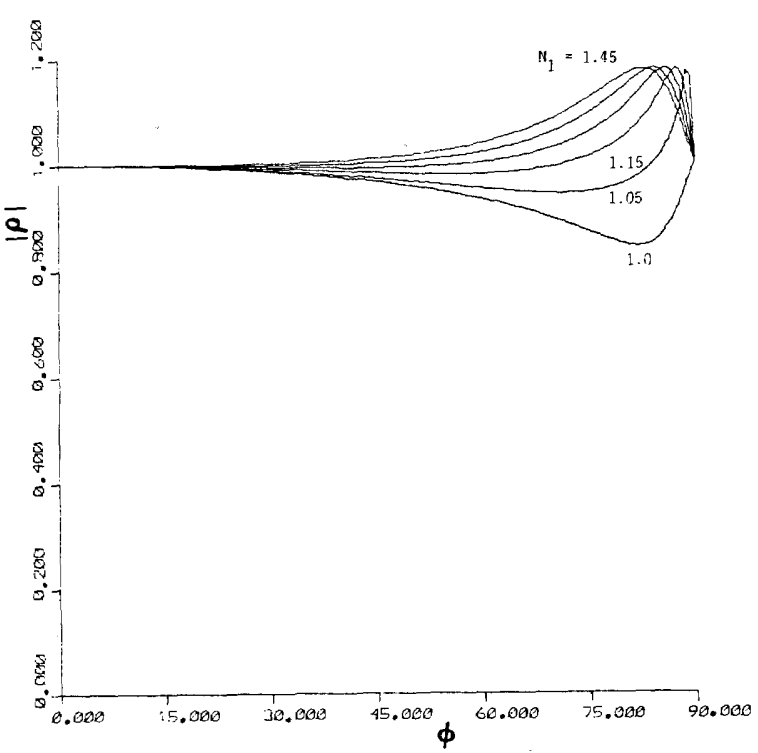

(a)

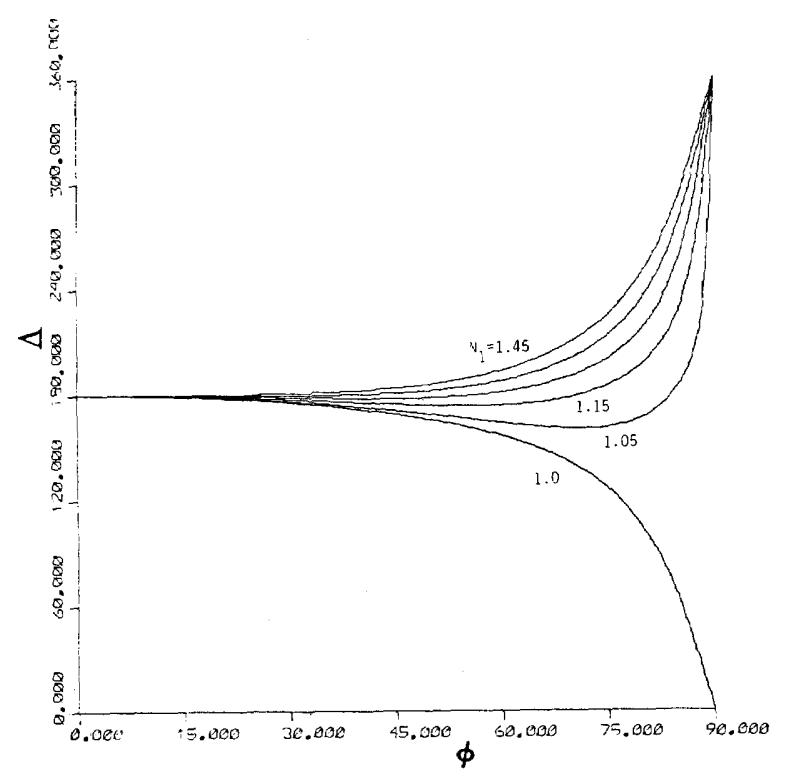

(b)

Fig. 8. (a) $|\rho|$ versus $\phi$ and (b) $\Delta$ versus $\phi$ for several transparentfilm-Al HWR's assuming different film refractive indices from 1.05 to 1.45 in equal steps of 0.1 . The curves marked 1.0 are those for the bare $\mathrm{Al}$ substrate.

metallic substrate. Each surface acts as a HWR, so that the net effect on polarization after two reflections at the same angle of incidence is null. We think that this is one of the simplest possible ways to make polarization-preserving beam displacers or axicons. This design represents an important special case of a more general one ${ }^{18,19}$ in which the same film-substrate system is used for each mirror or cone but the film thicknesses are unequal. Indeed, we were led to this work on HWR's by our earlier study of Refs. 18 and 19.

The film-substrate HWR also has direct application in equalization of the $p$ and $s$ (TE and TM) eigenvalues of $90^{\circ}$ rooftop reflectors and waxicons, so that these devices may be rendered isotropic when used in laser cavities. In this case it is assumed that the two reflections from a rooftop or a waxicon occur at the same angle of incidence of $45^{\circ}$.

\section{SUMMARY}

A system composed of a transparent film on an absorbing (or transparent) substrate can act as a single-reflection HWR at a given wavelength provided that the film refractive index $N_{1}$,
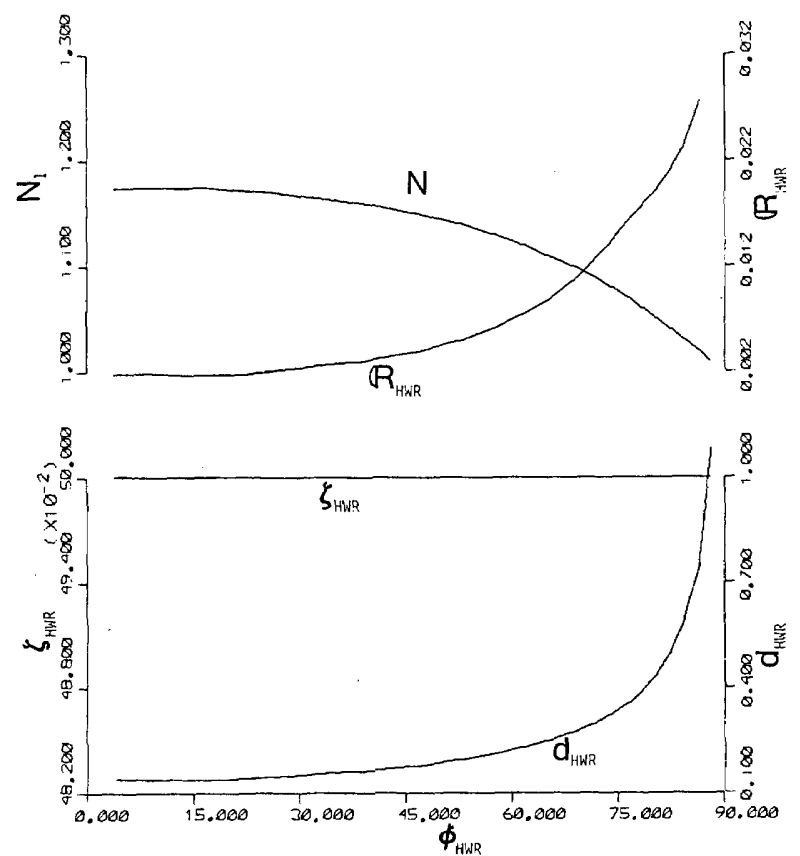

Fig. 9. Comprehensive results for $0.6328-\mu \mathrm{m}$ HWR's that use transparent films on a glass substrate of refractive index $N_{2}=1.5$. Top: film refractive index $N_{1}$ and intensity reflectance $\mathcal{R}_{\mathrm{HWR}}$. Bottom: normalized and actual least film thicknesses $\zeta_{\text {HWR }}$ and $d_{\text {HWR }}$ (in micrometers), respectively. The common abscissa for all curves is the angle of incidence $\phi_{\mathrm{HWR}}$ in degrees.
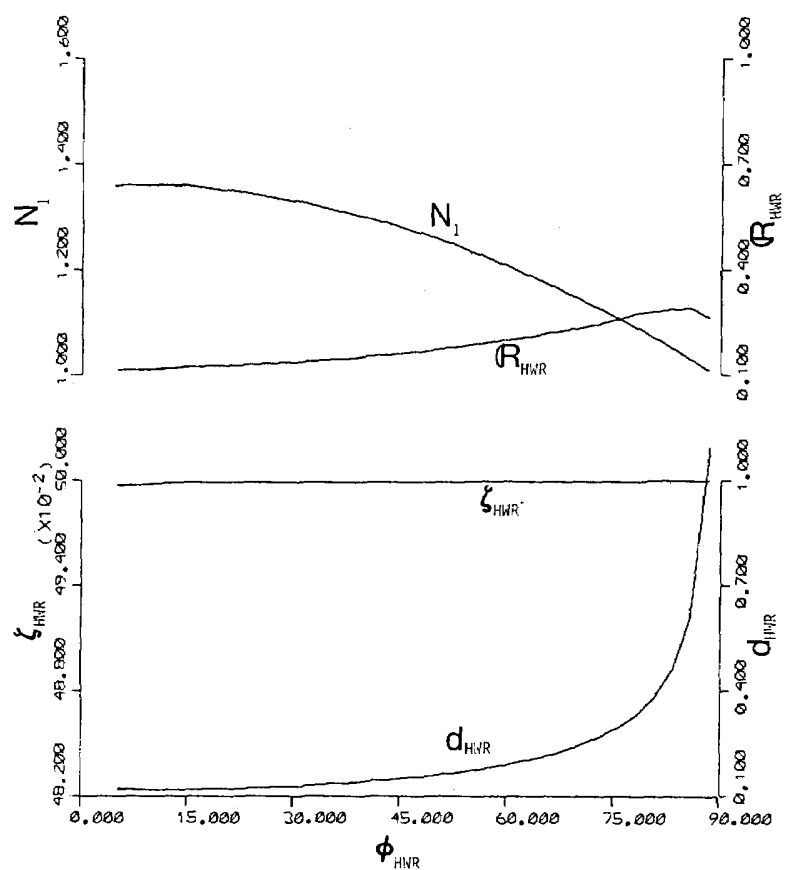

Fig. 10. Comprehensive results for $0.6328-\mu \mathrm{m}$ HWR's that use transparent films on a Si substrate of cumplex refractive index $\mathrm{N}_{2}=$ $3.85-j 0.02$. Top: film refractive index $N_{1}$ and intensity reflectance $\mathscr{R}_{\text {HWR }}$. Bottom: normalized and actual least film thicknesses $\zeta_{\text {HWR }}$ and $d_{\text {HWR }}$ (in micrometers), respectively. The common abscissa for all curves is the angle of incidence $\phi_{\mathrm{HWR}}$ in degrees. 

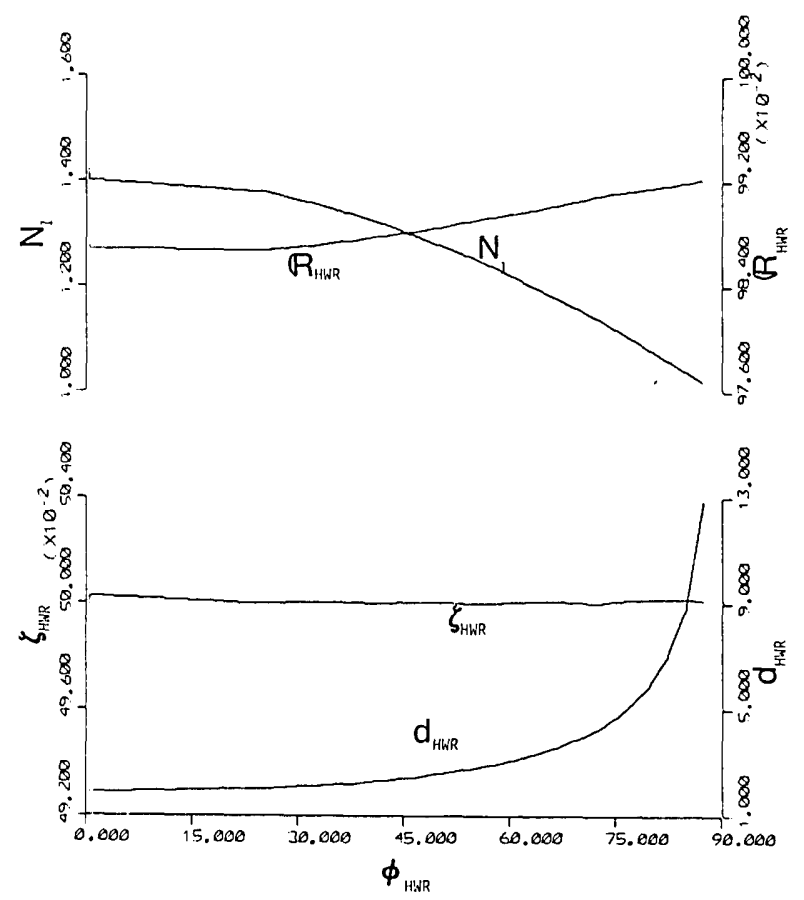

Fig. 11. Comprehensive results for 10.6- $\mu \mathrm{m}$ HWR's that use transparent films on an $\mathrm{Ag}$ substrate of complex refractive index $N_{2}=9.5$ - j73. Top: film refractive index $N_{1}$ and intensity reflectance $R_{\text {HWR }}$. Bottom: normalized and actual least film thicknesses $\zeta_{\text {HWR }}$ and $d_{\text {HWR }}$ (in micrometers), respectively. The common abscissa for all curves is the angle of incidence $\phi_{\mathrm{HWR}}$ in degrees.

film thickness $d$, and angle of incidence $\phi$ are properly selected. We find that a HWR is possible only within a certain interval of $N_{1}, 1<N_{1}<\hat{N}_{1}$, where $\hat{N}_{1}$ depends on the substrate and medium of incidence (which we take in this paper to be air). For an assumed $N_{1}$ within this range, a method is described (Section 2) that determines the angle of incidence $\phi_{\text {HWR }}$ and the least film thickness $d_{\text {HWR }}$ that produce halfwave retardation. We find that, as $N_{1}$ increases from 1 to $\hat{N}_{1}$, $\phi_{\text {HWR }}$ decreases monotonically from $90^{\circ}$ to 0 , respectively. $d_{\mathrm{HWR}} \approx 0.5 D_{\phi \mathrm{HWR}}$, where $D_{\phi}$ is the film-thickness period, and the approximately equal relationship is replaced by an exact equality in the limiting cases when the substrate is a perfect dielectric or a perfect conductor. Significantly, we find that all reflection characteristics of a film-substrate HWR remain close to their normal-incidence values as $\phi$ is increased from 0 to (and beyond) $\phi_{\text {HWR }}$. Thus these film-coated surfaces act as angle-insensitive reflectors.

Detailed results are given in Sections 2 and 4 for HWR's that consist of transparent films on metallic ( $\mathrm{Al}$ ), semiconducting (Si), and dielectric (glass) substrates at the $\mathrm{He}-\mathrm{Ne}$ laser wavelength $\lambda=0.6328 \mu \mathrm{m}$. Transparent film-on-silver HWR's that operate at the infrared $\mathrm{CO}_{2}$-laser wavelength $\lambda$ $=10.6 \mu \mathrm{m}$ are also designed. Only when the substrate is metallic does the device have the prerequisite high reflectance.

Film-substrate HWR's permit the realization of simple polarization-preserving beam displacers and axicons as well as isotropic (polarization-insensitive) $90^{\circ}$ rooftop reflectors and waxicons.
In this paper we have assumed the $e^{j \omega t}$ time dependence and $p$ and $s$ reference axes in the incident and reflected beams that are in accordance with the Nebraska (Muller) conventions. ${ }^{20}$

\section{ACKNOWLEDGMENT}

R. M. A. Azzam is pleased to acknowledge support by the National Science Foundation under grant no. DMR8018417.

* Present address, Intel Corporation, 3065 Bowers Avenue, Santa Clara, Calfornia 95051.

\section{REFERENCES}

1. This definition can be extended to circular and elliptical orthogonal polarization states (see Refs. 2-4). However, a circular phase retarder functions as a rotator, and elliptical retarders are uncommon.

2. W. A. Shurcliff, Polarized Light (Harvard U. Press, Cambridge, Mass., 1962).

3. R. M. A. Azzam and N. M. Bashara, Ellipsometry and Polarized Light (North-Holland, Amsterdam, 1977).

4. J. M. Bennett and H. E. Bennett, in Handbook of Optics, W. G. Driscoll and W. Vaughan, eds. (McGraw-Hill, New York, 1978), Chap. 10.

5. R. M. A. Azzam, A.-R. M. Zaghloul, and N. M. Bashara, "Ellipsometric function of a film-substrate system: applications to the design of reflection-type optical devices and to ellipsometry," J. Opt. Soc. Am. 65, 252-260 (1975).

6. A.-R. M. Zaghloul, R. M. A. Azzam, and N. M. Bashara, "Design of film-substrate single-reflection retarders," J. Opt. Soc. Am. 65, 1043-1049 (1975).

7. A.-R. M. Zaghloul, R. M. A. Azzam, and N. M. Bashara, "An angle-of-incidence tunable, $\mathrm{SiO}_{2}-\mathrm{Si}$ (film-substrate) reflection retarder for the UV mercury line $\lambda=2537 \AA$," Opt. Commun. 14, 260-262 (1975).

8. A.-R. M. Zaghloul, R. M. A. Azzam, and N. M. Bashara, " $\mathrm{SiO}_{2}-\mathrm{Si}$ film-substrate reflection retarders for different mercury spectral lines," Opt. Eng. 17, 180-184 (1978).

9. S. Kawabata and M. Suzuki, " $\mathrm{MgF}_{2}-\mathrm{Ag}$ tunable reflection retarder," Appl. Opt. 19, 484-485 (1980).

10. W. H. Southwell, "Multilayer coatings producing $90^{\circ}$ phase change," Appl. Opt. 18, 1875 (1979).

11. J. H. Apfel, "Graphical method to design multilayer phase retarders," Appl. Opt. 20, 1024-1029 (1981).

12. J. H. Apfel, "Phase retardance of periodic multilayer mirrors," Appl. Opt. 21, 733-738 (1982).

13. A discussion of this property and its applications can be found in a recent note: R. M. A. Azzam, "The change of polarization that accompanies normal-incidence reflection from a passive isotropic surface," Optik (to be published).

14. T. H. Allen, "Study of Al with combined Auger electron spectrometer-ellipsometer system," J. Vac. Sci. Technol. 13, 112-115 (1976).

15. G. Hass, in Applied Optics and Optical Engineering, R. Kingslake, ed. (Academic, New York, 1965), Vol. III, Chap. 8.

16. This happens to be the refractive index of $\mathrm{SiO}_{2}$ at $\lambda=0.6328$ $\mu \mathrm{m}$.

17. W. L. Wolfe, in Handbook of Optics, W. G. Driscoll and W. Vaughan, eds. (McGraw-Hill, New York, 1978), Chap. 7.

18. R. M. A. Azzam, "Displacement of a monochromatic light beam parallel to itself without change of polarization," Opt. Lett. 7, 80-82 (1982).

19. R. M. A. Azzam and M. Emdadur Rahman Khan, "Polarization-preserving single-layer-coated beam displacers and axicons," Appl. Opt. 21, 3314-3322 (1982).

20. R. H. Muller, "Definitions and conventions in ellipsometry," Surf. Sci. 16, 14-33 (1969). 